\title{
The thermal and mechanical behaviour of structural steel piping systems
}

\author{
E.M.M. Fonseca ${ }^{\text {a,* }}$, F.J.M.Q. de Melo ${ }^{\text {b }}$, C.A.M. Oliveira ${ }^{c}$ \\ ${ }^{a}$ Department of Applied Mechanics, Polytechnic Institute of Bragança-ESTIG, Campus De Sta Apolonia, Apartado 1134, Bragança 5301 857, Portugal \\ ${ }^{\mathrm{b}}$ Department of Mechanical Engineering, University of Aveiro, Aveiro, Portugal \\ ${ }^{\mathrm{c}}$ Department of Mechanical Engineering and Industrial Management, Faculty of Engineering of University of Porto, Porto, Portugal
}

Received 1 October 2003; revised 28 June 2004; accepted 28 June 2004

Abstract

The temperature, the deformation and the stress field in thermo-mechanical problems play a very important role in engineering applications. This paper presents a finite element algorithm developed to perform the thermal and mechanical analysis of structural steel piping systems subjected to elevated temperatures. The new pipe element with 22 degrees of freedom has a displacement field that results from the superposition of a beam displacement, with the displacement field associated with the section distortion. Having determined the temperature field, the consequent thermal displacement produced in the piping systems due to the thermal variation can be calculated. The temperature rise produces thermal expansion and a consequent increase of pipe length in the structural elements. For small values of the ratio of the pipe thickness to mean radius, the thermal behaviour can be calculated with adequate precision using a one-dimensional mesh approach, with thermal boundary conditions of an axisymmetric type across the pipe section. With this condition, several case studies of piping systems subjected to elevated temperatures and mechanical loads are presented and compared with corresponding results from commercial finite element codes. The main advantage of this formulation is associated with reduced time for mesh generation with a low number of elements and nodes. Considerable computational effort may be saved with the use of this finite pipe element.

(C) 2004 Elsevier Ltd. All rights reserved.

Keywords: Piping system; Elevated temperatures; Thermo-mechanical analysis; Finite pipe element

\section{Introduction}

Structural piping systems are widely used in industrial plants. These structures are not only subjected to mechanical loads, but are frequently exposed to aggressive thermal loads. The complexity of the analysis of such systems needs powerful numerical techniques with high computing performance, which is the case of the finite element analysis here described.

Neglecting the heat generated due to mechanical deformation, the thermal and the mechanical problems are uncoupled. The technique involves simultaneously solving an uncoupled set of equations, the transient heat conduction equation and the incremental equilibrium equation,

\footnotetext{
* Corresponding author. Tel.: +351-273-303-155; fax: +351-273313-051.

E-mail address: efonseca@ipb.pt (E.M.M. Fonseca).
}

performed for each time interval in the incremental solution. The same finite element formulation (finite element mesh, shape function, etc.) and the same equation solution technique (frontal solution technique) are used both for the thermal and the stress analysis. The thermal model is presented in Ref. [1] using a differential heat conduction formulation, which permits the calculation of the temperature field. The mechanical model developed for tubular structures will be presented in this paper, using elasticity theory for a new finite pipe element with two nodes that is superimposed with Fourier series for warping and ovalization displacement fields.

\section{Thermal and mechanical analysis formulation}

The deformation field of a pipe element refers to membrane strain and curvature variation. The geometric parameters considered for this element definition are: the arc 
(a)

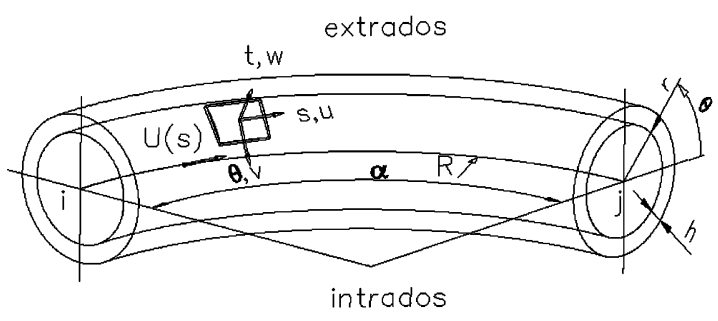

(b)

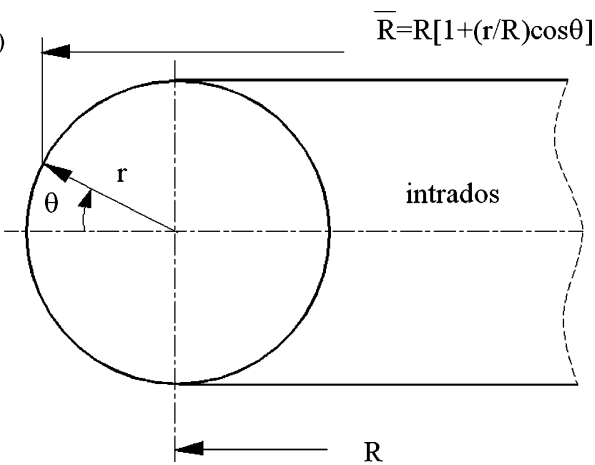

169

170

171

172

173

174

175

176

Fig. 1. (a) Geometric parameters for the finite pipe element. (b) The radius of curvature for the curved pipe. length $(s)$, the mean radius of curvature $(R)$, the thickness $(h)$, the mean section radius of the pipe $(r)$ and the central angle $(\alpha)$. Fig. 1a shows the essential geometric parameters defining the two-node finite pipe element. The basic equations governing the behaviour of thin shells were derived by Love [2]. The following assumptions referred to in Refs. [3-5], were considered in the present analysis:

- the radius of curvature is assumed much larger then the section radius. This means that the 'pipe bore term' $(R+r \cos \theta)$ may be approximated to $R$, Fig. 1b;

- a semi-membrane deformation model is adopted and neglects the bending stiffness along the longitudinal direction of the toroidal shell, but considers the meridional bending resulting from ovalization;

- the shell is considered thin and inextensible along the meridional direction in the mechanical case. Typically, the thickness should be less than a tenth of the mean radius of the pipe.

In the mechanical model, having assumed small strains, the complete incremental relation between stress and strain for thermal and mechanical deformation is found to be

$\varepsilon=\varepsilon^{\mathrm{mec}}+\varepsilon^{\text {th }}$

where $\varepsilon^{\text {mec }}$ is the mechanical strain increment and $\varepsilon^{\text {th }}$ the thermal strain.

The application of the principle of virtual work gives finally the system of algebraic equations to be solved. The matrix force-displacement equation for this finite pipe element model is

$$
[K]\{\delta\}=\left\{F_{\mathrm{n}}\right\}+\left\{F_{\mathrm{th}}\right\}
$$

where $\{\delta\}$ is a nodal unknown displacement vector, $\left\{F_{\mathrm{n}}\right\}$ is the applied nodal forces and $\left\{F_{\text {th }}\right\}$ is a nodal force vector due to thermal effects. The element stiffness matrix $[K]$ is calculated from the matrix equation:

$K=\int_{s=0}^{s=L} \int_{\theta=0}^{\theta=2 \pi}[B]^{\mathrm{T}}[D][B] r \mathrm{~d} s \mathrm{~d} \theta$
The following expression represents the nodal forces due to the thermal strain

$F_{\text {th }}=\int_{V}[B]^{\mathrm{T}}[D]\{\varepsilon\}^{\text {th }} \mathrm{d} V$

where $\mathrm{d} V$ is the elementary pipe volume, $[D]$ the elasticity matrix and $[B]$ results from the derivative of the shape functions for the pipe element.

Having solved the system of algebraic equations, the displacement field is calculated for all the nodes of the structure. The stress field is then defined for each element in the following form

$\sigma=[D]\left(\{\varepsilon\}^{\mathrm{mec}}-\{\varepsilon\}^{\mathrm{th}}\right)+\{\sigma\}_{0}$

where $\sigma_{0}$ represents the initial stresses.

The elasticity matrix $[D]$ appears with a simple algebraic definition, where the off-diagonal terms vanish

$D=\left[\begin{array}{ccc}\frac{E h}{1-\nu^{2}} & 0 & 0 \\ 0 & \frac{E h}{2(1+\nu)} & 0 \\ 0 & 0 & \frac{E h^{3}}{12\left(1-\nu^{2}\right)}\end{array}\right]$

where $E$ is the elastic modulus that is a temperature dependent parameter, according to Eurocode 3 [6], $h$ is the pipe thickness and $\nu$ is Poisson's ratio.

\section{The displacement field for a new two-node pipe element}

The displacements $u, v$ and $w$ are calculated for the shell surface from the structural element. These functions refer to the displacement field on the mean line arc 

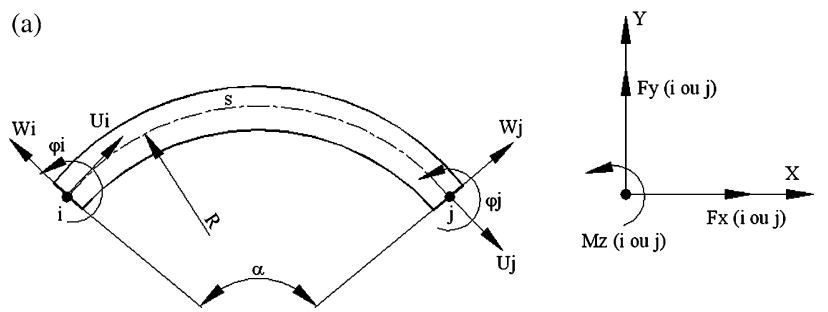

(b)

Fig. 2. (a) Degrees of freedom for in-plane element. (b) Degrees of freedom for out-of-plane element.

$(U, W$ and $\varphi)$. Those parameters are related via simple differential equations from beam bending theory, following simple hypotheses considered by Melo and Castro [7,8] and Thomson [5]

$$
\begin{aligned}
& \varphi=\frac{\mathrm{d} W}{\mathrm{~d} s} \\
& W=-\frac{\mathrm{d} U}{\mathrm{~d} s} R
\end{aligned}
$$

The displacement field is calculated from the mean line of each arc considered like a rigid beam element: $U$ is the tangential displacement, $W$ and $\bar{W}$ the transverse displacements, $\beta, \bar{\varphi}$ and $\varphi$ are the rotations in each direction represented in Fig. 2a and b, respectively, for in-plane or out-of-plane loading.

When the finite pipe element has in-plane displacements, a high-order formulation is used and six parameters are necessary to define the displacement field. So, $U$ can be approximated by the following fifth-order polynomial

$U_{(s)}=a_{0}+a_{1} s+a_{2} s^{2}+a_{3} s^{3}+a_{4} s^{4}+a_{5} s^{5}$

The coefficients in Eq. (9) are determined by imposing boundary conditions according to the curved reference. With those specified conditions, the functions of the generic local displacements for an in-plane element (IN) are given by the following equations:

$$
\begin{aligned}
U_{(s) \mathrm{IN}}= & \left(U_{i} N_{u i}+U_{j} N_{u j}\right)+\left(W_{i} N_{w i}+W_{j} N_{w j}\right) \\
& +\left(\varphi_{i} N_{\varphi i}+\varphi_{j} N_{\varphi j}\right) \\
W_{(s) I \mathrm{~N}}= & -R\left(\left(U_{i} N_{u i}^{\prime}+U_{j} N_{u j}^{\prime}\right)+\left(W_{i} N_{w i}^{\prime}+W_{j} N_{w j}^{\prime}\right)\right. \\
& \left.+\left(\varphi_{i} N_{\varphi i}^{\prime}+\varphi_{j} N_{\varphi j}^{\prime}\right)\right) \\
\varphi_{(s) \mathrm{IN}}= & -R\left(\left(U_{i} N_{u i}^{\prime \prime}+U_{j} N_{u j}^{\prime \prime}\right)+\left(W_{i} N_{w i}^{\prime \prime}+W_{j} N_{w j}^{\prime \prime}\right)\right. \\
& \left.+\left(\varphi_{i} N_{\varphi i}^{\prime \prime}+\varphi_{j} N_{\varphi j}^{\prime \prime}\right)\right)
\end{aligned}
$$

The shape functions are determined as follows:

$$
\begin{aligned}
N_{u i}= & \cos \left(\frac{\alpha}{2}\right)+\frac{1}{R} \sin \left(\frac{\alpha}{2}\right) s \\
& +\left(-\frac{10}{L^{3}} \cos \left(\frac{\alpha}{2}\right)-\frac{6}{R L^{2}} \sin \left(\frac{\alpha}{2}\right)\right) s^{3} \\
& +\left(\frac{15}{L^{4}} \cos \left(\frac{\alpha}{2}\right)+\frac{8}{R L^{3}} \sin \left(\frac{\alpha}{2}\right)\right) s^{4} \\
& +\left(-\frac{6}{L^{5}} \cos \left(\frac{\alpha}{2}\right)-\frac{3}{R L^{4}} \sin \left(\frac{\alpha}{2}\right)\right) s^{5}
\end{aligned}
$$

$$
\begin{aligned}
N_{u j}= & \left(\frac{10}{L^{3}} \cos \left(\frac{\alpha}{2}\right)+\frac{4}{R L^{2}} \sin \left(\frac{\alpha}{2}\right)\right) s^{3} \\
& +\left(-\frac{15}{L^{4}} \cos \left(\frac{\alpha}{2}\right)-\frac{7}{R L^{3}} \sin \left(\frac{\alpha}{2}\right)\right) s^{4} \\
& +\left(\frac{6}{L^{5}} \cos \left(\frac{\alpha}{2}\right)+\frac{3}{R L^{4}} \sin \left(\frac{\alpha}{2}\right)\right) s^{5}
\end{aligned}
$$

$$
\begin{aligned}
N_{w i}= & \sin \left(\frac{\alpha}{2}\right)-\frac{1}{R} \cos \left(\frac{\alpha}{2}\right) s \\
& +\left(-\frac{10}{L^{3}} \sin \left(\frac{\alpha}{2}\right)+\frac{6}{R L^{2}} \cos \left(\frac{\alpha}{2}\right)\right) s^{3} \\
& +\left(\frac{15}{L^{4}} \sin \left(\frac{\alpha}{2}\right)-\frac{8}{R L^{3}} \cos \left(\frac{\alpha}{2}\right)\right) s^{4} \\
& +\left(-\frac{6}{L^{5}} \sin \left(\frac{\alpha}{2}\right)+\frac{3}{R L^{4}} \cos \left(\frac{\alpha}{2}\right)\right) s^{5}
\end{aligned}
$$

$$
\begin{aligned}
N_{w j}= & \left(-\frac{10}{L^{3}} \sin \left(\frac{\alpha}{2}\right)+\frac{4}{R L^{2}} \cos \left(\frac{\alpha}{2}\right)\right) s^{3} \\
& +\left(\frac{15}{L^{4}} \sin \left(\frac{\alpha}{2}\right)-\frac{7}{R L^{3}} \cos \left(\frac{\alpha}{2}\right)\right) s^{4} \\
& +\left(-\frac{6}{L^{5}} \sin \left(\frac{\alpha}{2}\right)+\frac{3}{R L^{4}} \cos \left(\frac{\alpha}{2}\right)\right) s^{5}
\end{aligned}
$$

$$
N_{\varphi i}=-\frac{1}{2 R} s^{2}+\frac{3}{2 L R} s^{3}-\frac{3}{2 L^{2} R} s^{4}+\frac{1}{2 L^{3} R} s^{5}
$$


$N_{\varphi j}=-\frac{1}{2 L R} s^{3}+\frac{1}{L^{2} R} s^{4}-\frac{1}{2 L^{3} R} s^{5}$

The displacement field out-of-plane, designated as OUT, in a local reference system, is determined with the following equations:

$W_{(s) \text { OUT }}=\bar{W}_{i} N_{1}-\bar{\varphi}_{i} N_{2}+\bar{W}_{j} N_{3}-\bar{\varphi}_{j} N_{4}$

$\varphi_{(s) \mathrm{OUT}}=\bar{W}_{i} N_{1}^{\prime}-\bar{\varphi}_{i} N_{2}^{\prime}+\bar{W}_{j} N_{3}^{\prime}-\bar{\varphi}_{j} N_{4}^{\prime}$

$\beta_{(s) \mathrm{OUT}}=\beta_{i} N_{i}+\beta_{j} N_{j}$

The shape functions used in Eqs. (12a)-(12c) are thirdorder polynomials $\left(N_{1}, N_{2}, N_{3}, N_{4}\right)$ and first-order polynomials $\left(N_{i}, N_{j}\right)$, respectively.

The surface displacement in the radial direction results from ovalization, in-plane and out-of-plane, from Ref. [5] and is expressed by the equation:

$$
\begin{aligned}
w(s, \theta)= & \left(\sum_{n \geq 2} a_{n} \cos n \theta+\sum_{n \geq 2} \bar{a}_{n} \sin n \theta\right) N_{i} \\
& +\left(\sum_{n \geq 2} a_{n} \cos n \theta+\sum_{n \geq 2} \bar{a}_{n} \sin n \theta\right) N_{j}
\end{aligned}
$$

The meridional displacement due to ovalization is calculated from the following equation:

$$
\begin{aligned}
v(s, \theta)= & \left(-\sum_{n \geq 2} \frac{a_{n}}{n} \sin n \theta+\sum_{n \geq 2} \frac{\bar{a}_{n}}{n} \cos n \theta\right) N_{i} \\
& +\left(-\sum_{n \geq 2} \frac{a_{n}}{n} \sin n \theta+\sum_{n \geq 2} \frac{\bar{a}_{n}}{n} \cos n \theta\right) N_{j}
\end{aligned}
$$

Finally, the longitudinal displacement due to warping of the pipe section is defined from the following equation:

$$
\begin{aligned}
u(s, \theta)= & \left(\sum_{n \geq 2} b_{n} \cos n \theta+\sum_{n \geq 2} \bar{b}_{n} \sin n \theta\right) N_{i} \\
& +\left(\sum_{n \geq 2} b_{n} \cos n \theta+\sum_{n \geq 2} \bar{b}_{n} \sin n \theta\right) N_{j}
\end{aligned}
$$

The terms $a_{n}$ and $\bar{a}_{n}$ are constants to be determined and included in the Fourier expansions for the ovalization displacements of in-plane and out-of-plane bending, respectively. The parameters $b_{n}$ and $\bar{b}_{n}$ are also functions of developed series resulting from warping displacements in and out-of-plane.

When a tubular system without restraint is subjected to temperature variation, there will be a length increase and the temperature produces dilation along the cross-section of the pipe. The perimeter of the pipe will be variable. Finally, following the proposed formulation, the finite shell element displacement field, resulting from the superposition of the displacement of a rigid beam and from the complete Fourier expansion for ovalization and warping terms in Eqs. (13)-(15), leads to:

$u=U_{(s) \mathrm{IN}}-r \cos \theta \varphi_{(s) \mathrm{IN}}-r \sin \theta \varphi_{(s) \mathrm{OUT}}+u(s, \theta)+s \bar{\alpha} \Delta T$

$v=-W_{(s) \mathrm{IN}} \sin \theta+W_{(s) \mathrm{OUT}} \cos \theta+r \beta_{(s) \mathrm{OUT}}+v(s, \theta)$

$w=W_{(s) \mathrm{IN}} \cos \theta+W_{(s) \mathrm{OUT}} \sin \theta+w(s, \theta)+r \bar{\alpha} \Delta T$

The displacement field of the shell surface in a condensed vector representation is

$$
\begin{aligned}
\left\{\begin{array}{c}
u \\
v \\
w
\end{array}\right\} & =\left\{\begin{array}{c}
u \\
v \\
w
\end{array}\right\}_{\mathrm{mec}}+\left\{\begin{array}{c}
u \\
v \\
w
\end{array}\right\}_{\mathrm{th}} \\
& =[N] \times\{\delta\}+\left\{\begin{array}{c}
s \bar{\alpha} \Delta T \\
0 \\
r \bar{\alpha} \Delta T
\end{array}\right\}
\end{aligned}
$$

where $\bar{\alpha}$ is the thermal expansion coefficient, considered constant in this formulation and $\Delta T$ is the temperature variation.

As referred to previously, the mechanical deformation model considers that the pipe undergoes a semi-membrane strain field. The strain field is given by the following equations also used by Melo and Castro [7,8], Flügge [9] and Kitching [10]

$$
\begin{aligned}
\underset{\sim \mathrm{mec}}{\varepsilon}= & \left\{\begin{array}{c}
\varepsilon_{s s} \\
\gamma_{s \theta} \\
\chi_{\theta \theta}
\end{array}\right\} \\
= & {\left[\begin{array}{ccc}
\frac{\partial}{\partial s} & -\frac{\sin \theta}{R} & \frac{\cos \theta}{R} \\
\frac{1}{r} \frac{\partial}{\partial \theta} & \frac{\partial}{\partial s} & 0 \\
0 & -\frac{1}{r^{2}} \frac{\partial}{\partial \theta} & \frac{1}{r^{2}} \frac{\partial^{2}}{\partial \theta^{2}}
\end{array}\right]\left\{\begin{array}{c}
u \\
v \\
w
\end{array}\right\} }
\end{aligned}
$$

where $\varepsilon_{s s}$ is the longitudinal membrane strain, $\gamma_{s \theta}$ is the shear strain and $\chi_{\theta \theta}$ is the meridional curvature from ovalization.

The dimension of the deformation vector increases one term due to the thermal circumferential deformation by the expression:

$\varepsilon_{\theta \theta}=\frac{1}{r}\left(w+\frac{\partial v}{\partial \theta}\right)=\frac{r \bar{\alpha} \Delta T}{r}$

The thermal deformation vector is obtained from the following equation:

$$
\left\{\begin{array}{c}
\varepsilon_{s s} \\
\varepsilon_{\theta \theta}
\end{array}\right\}^{\text {th }}=\left\{\begin{array}{l}
\bar{\alpha} \Delta T \\
\bar{\alpha} \Delta T
\end{array}\right\}
$$




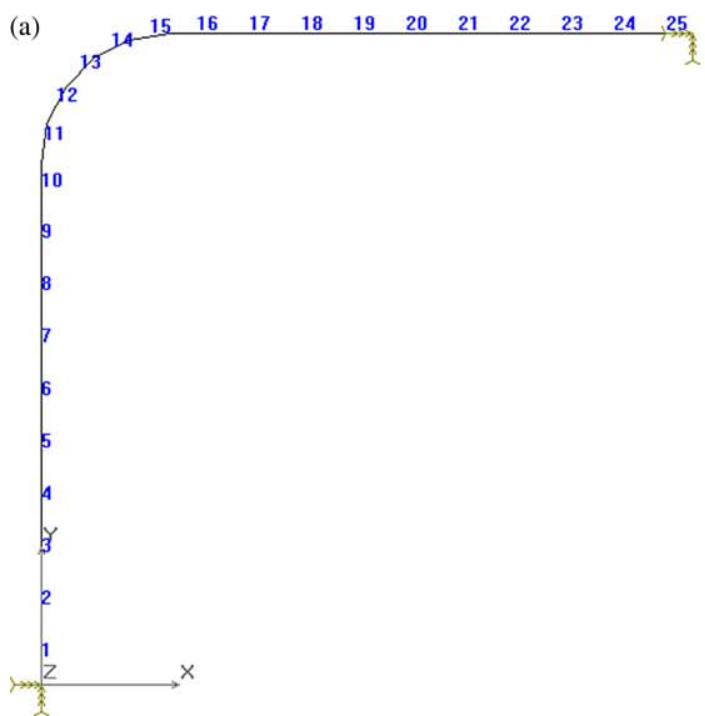

(b)

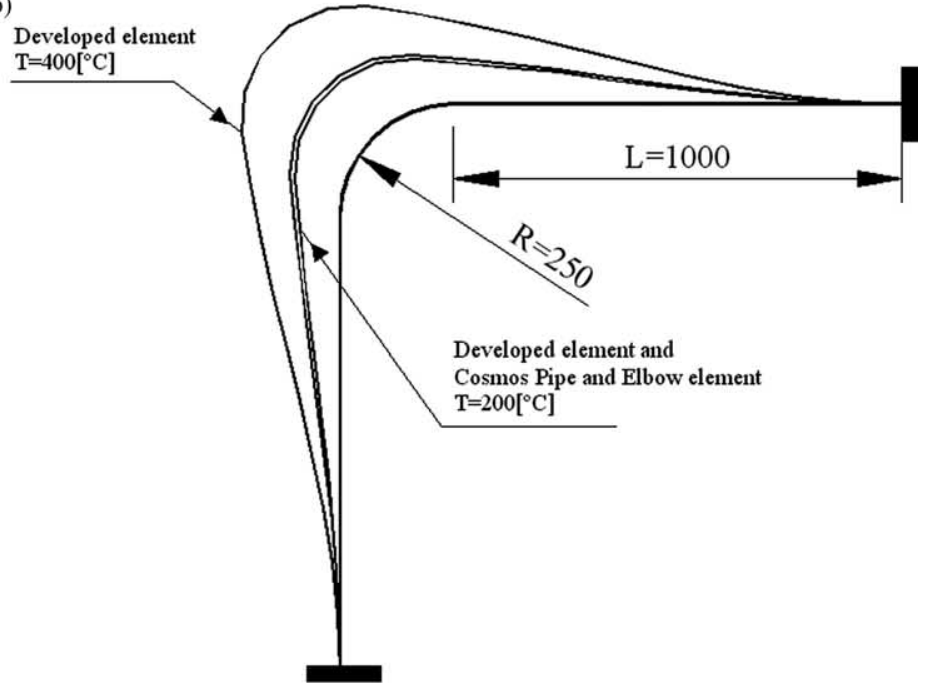

Fig. 3. (a) Finite element mesh used in developed program. (b) Displacement nodal results for different temperatures. Deformed shape magnified $30 \times$.

(a)

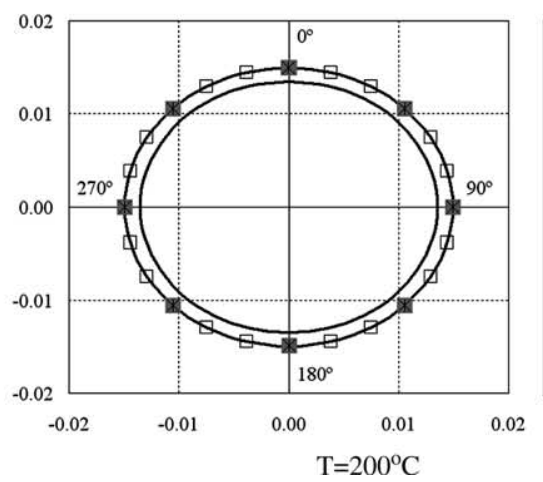

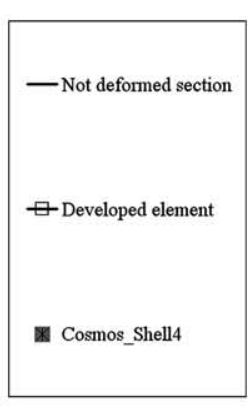

(b)

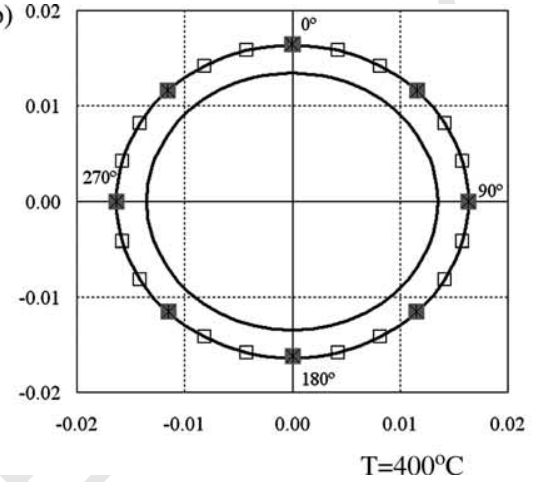

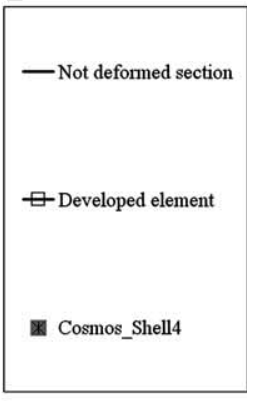

Tosmos_Shell4

(b)

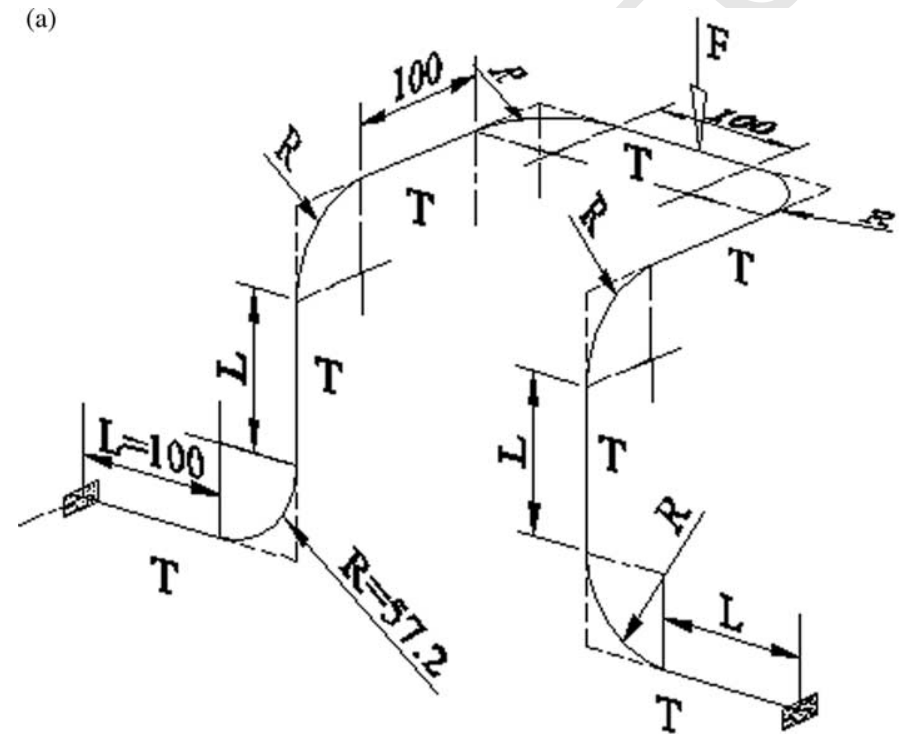

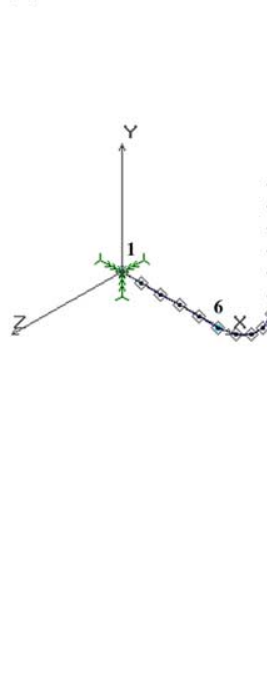

505

506

507

508

509

510

511

512

513

514

515

516

517

518

519

520

521

522

523

524

525

526

527

528

529

530

531

532

533

534

535

536

537

538

539

540

541

542

543

544

545

546

547

548

549

550

551

552

553

554

555

556

557

558

559

560 

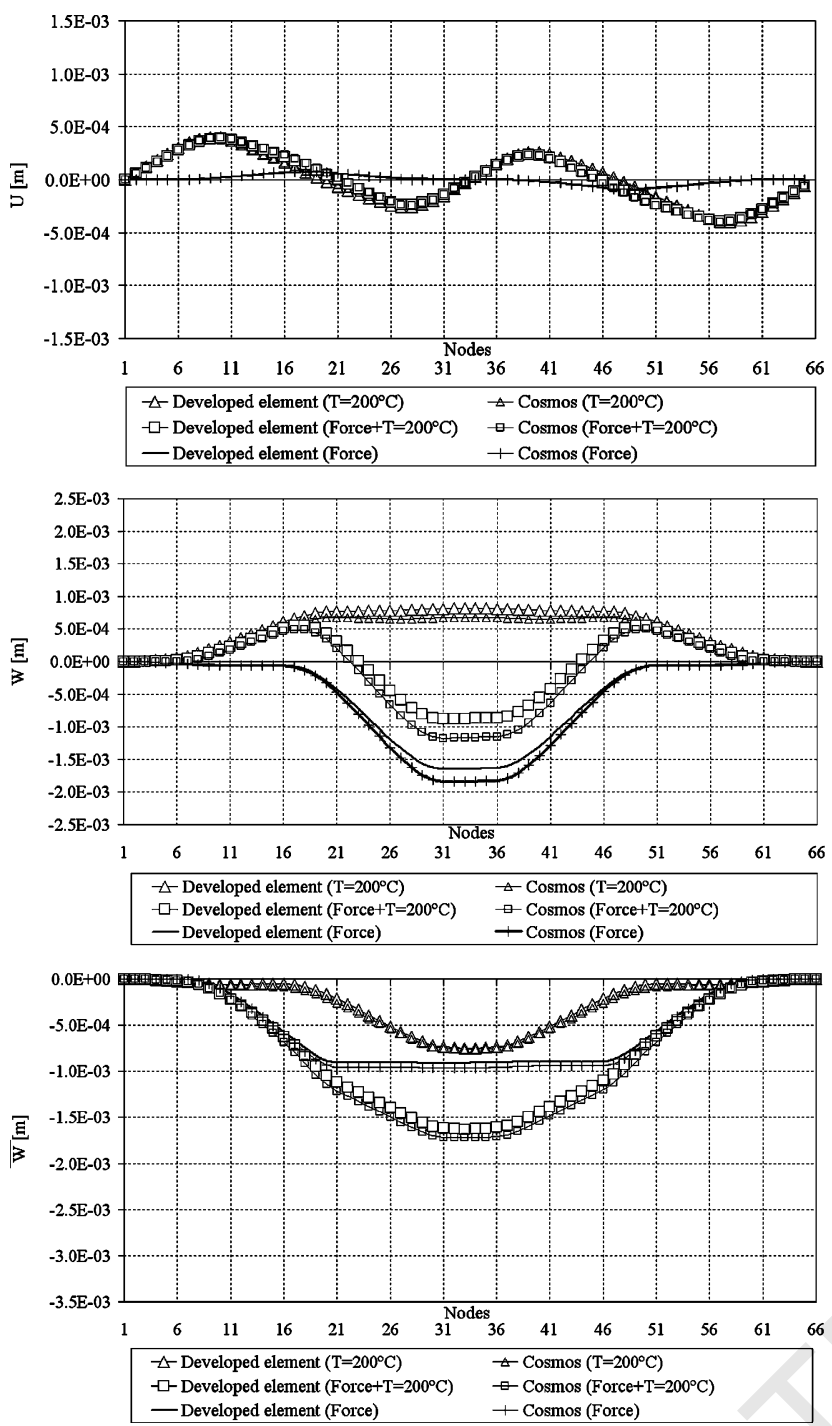

617

618

619

620

621

622

623
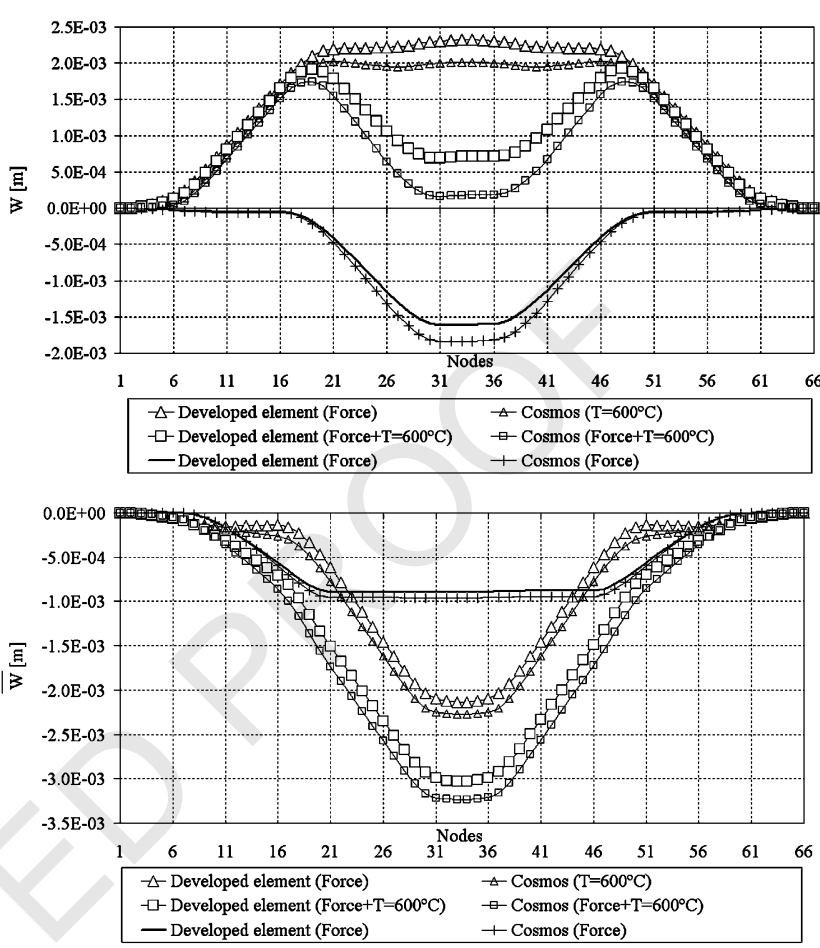

Fig. 6. Displacement fields obtained using the developed pipe element and the COSMOS program for Case 2.

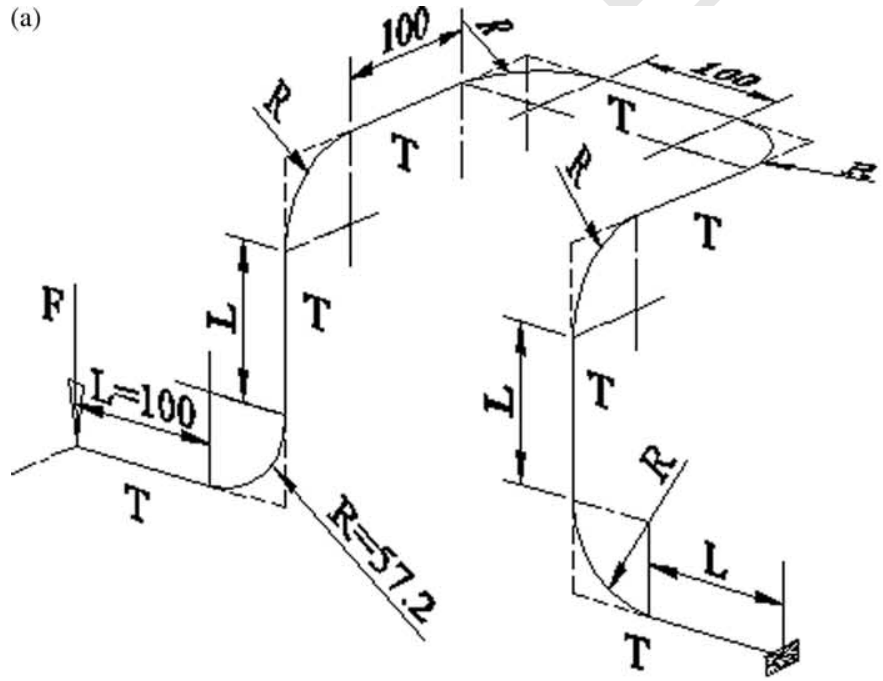

(b)

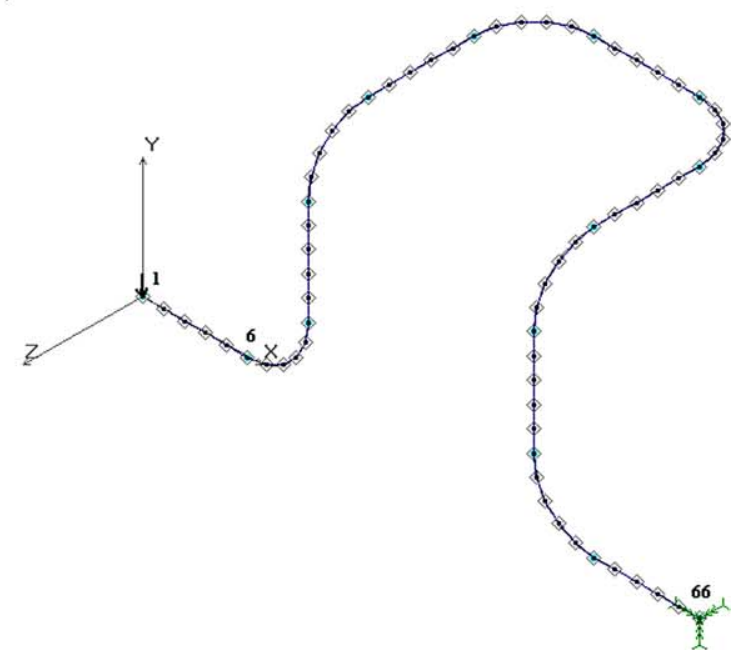

Fig. 7. (a) Geometry of piping system. (b) Mesh used and the thermal and mechanical boundary conditions. 

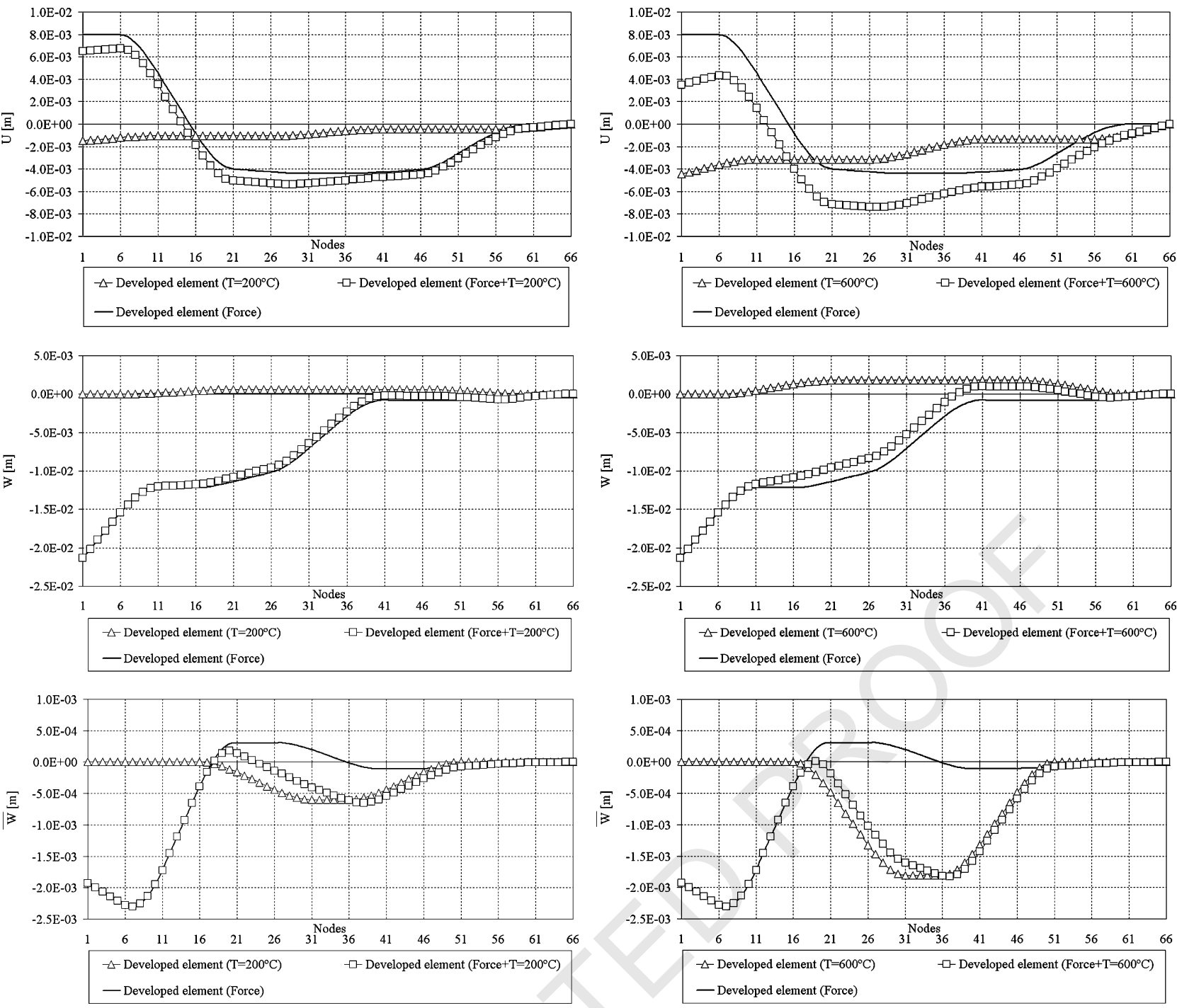

Fig. 8. Displacement fields obtained using the developed pipe element for Case 3.
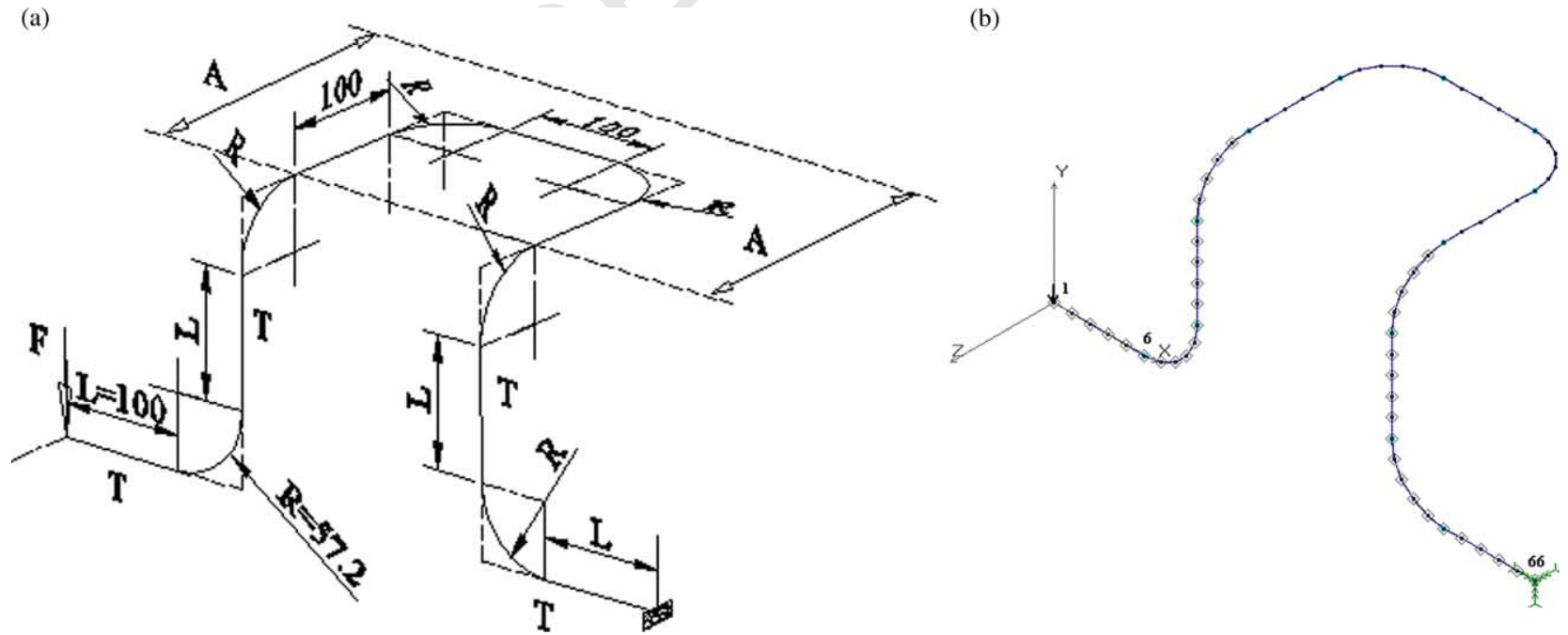

Fig. 9. (a) Geometry of piping system. (b) Mesh used and the thermal and mechanical boundary conditions. 

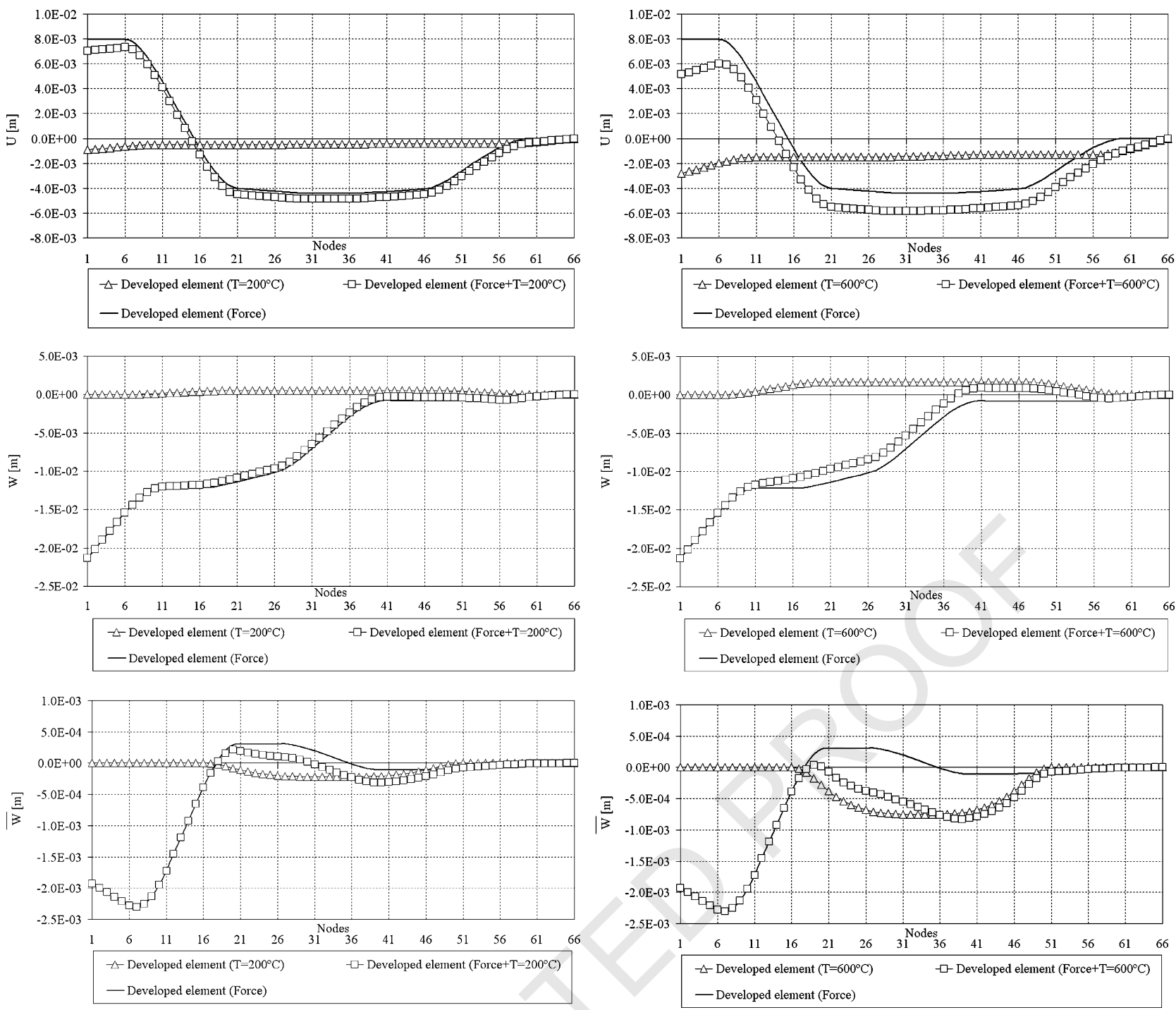

841

842

843

844

845

846

847

Fig. 10. Displacement fields obtained using the developed pipe element for Case 4.

\section{Case studies}

\subsection{Case 1: a plane geometry piping system subjected to elevated temperatures}

A tubular steel piping system has a radius of curvature equal $0.25 \mathrm{~m}$, a mean radius of $0.0135 \mathrm{~m}$ and the thickness is $0.001 \mathrm{~m}$, Fig. 3a. The piping system has end restraints and is subjected to different temperatures. The thermal expansion coefficient $\bar{\alpha}$ is constant and equal to $14 \times 10^{-6}{ }^{\circ} \mathrm{C}^{-1}$, $\nu=0.3$ and $E$ is a function of temperature, according to Eurocode3 [6].

The deformed shape obtained is magnified $30 \times$ in Fig. 3b. For a temperature rise of $T=200{ }^{\circ} \mathrm{C}$ numerical results have been compared with a commercial code COSMOS using Pipe and Elbow elements as shown in Fig. 3b.

Fig. 4 represents the transverse displacement obtained with different temperatures for a section in the tubular straight pipe for element 5. As can be observed, the pipe cross-section has the mean radius increased with the thermal expansion. The results obtained with the element developed herein are compared with the COSMOS program using a Shell element. This is an advantage with our code; it is possible with the same element to calculate the displacement field in the shell surface.

4.2. Case 2: a piping system with spatial geometry subjected to elevated temperatures having two end restraints and a vertical force at the mid-length of the structure

The next case is a structural steel piping system with all elements subjected to uniform temperature $(T=200$ or $600{ }^{\circ} \mathrm{C}$ ) and to a vertical force of $F=3000 \mathrm{~N}$, Fig. 5a. The structure also has end restraints. The system has a mean radius of $0.022 \mathrm{~m}$ and a thickness of $0.0025 \mathrm{~m}$. The thermal expansion coefficient is equal to $14 \times 10^{-6}{ }^{\circ} \mathrm{C}^{-1}$, $\nu=0.3$ and $E$ is a function of temperature, according to 
Eurocode3 [6]. The finite pipe element mesh uses 65 elements as represented in Fig. $5 b$.

The results are compared with those obtained using the COSMOS programme with Pipe and Elbow elements as shown in Fig. 6. We have also compared the influence of the temperature in the structure simultaneously with or without a vertical force.

\subsection{Case 3: a piping system with spatial geometry subjected to elevated temperatures having one end restraint and a vertical force on the other free end}

This case is the same as Case 2, but now with the spatial structure subjected to a vertical force, $F=3000 \mathrm{~N}$ at one end and the other end restrained, see Fig. 7a. The structure is subjected to two uniform different temperatures of $T=200$ and $600{ }^{\circ} \mathrm{C}$.

The transverse displacement field is influenced by the temperature rise, as shown in Fig. 8, having compared these results with those corresponding to the mechanical standalone load.

\subsection{Case 4: a piping system with spatial geometry subjected} to elevated temperatures with upper length zone insulated, having one end restrained and a vertical force on the other free end

The same piping system is now subjected to the same different boundary conditions (vertical force of $F=3000 \mathrm{~N}$ in free end and the other end restrained, partially subjected to uniform temperature $\left(T=200\right.$ or $\left.600{ }^{\circ} \mathrm{C}\right)$ and partially insulated in the upper length zone AA, see Fig. 9a.

The displacement field increases as can be seen in Fig. 10, compared with the results for Case 3.

\section{Conclusions}

The results for the structural displacements resulting from elevated temperatures and mechanical actions on tubular structures have been presented, using a new finite element for linear and elastic formulation. Good agreement between the displacement results obtained with the finite element presented here and corresponding data from other commercial codes was observed. This new two-node finite pipe element presents good agreement in the analysis of thermo-mechanical problems, with all shell membrane capabilities, when compared to other possible finite modelling techniques. It is possible to calculate the displacement field due to elevated temperatures. This finite element is easy to operate, demands small computer capacity and avoids the need to use expensive meshes for the shell surface definition.

\section{References}

[1] Fonseca EMM. Finite element analysis of structural piping systems behaviour under high thermal gradients, $\mathrm{PhD}$ thesis (in Portuguese), Faculty of Engineering of University of Porto, Porto; 2003.

[2] Love AEH. The mathematical theory of elasticity. New York: Dover; 1944.

[3] Madureira L, Melo FQ. A hybrid formulation in the stress analysis of curved pipes. Eng Comput 2000;17(8):970-80.

[4] Fonseca EMM, Melo FJMQ, Oliveira CAM. Determination of flexibility factors on curved pipes with end restraints using a semianalytic formulation. Int J Press Vessels Piping 2002;79/12:829-40.

[5] Thomson G. The influence of end constraints on pipe bends, $\mathrm{PhD}$ Thesis, University of Strathclyde, Scotland, UK; 1980.

[6] CEN ENV 1993-1-2, Eurocode 3: Design of steel structures-Part 1.2: general rules—structural fire design; 1995.

[7] Melo FJMQ, Castro PMST. A reduced integration Mindlin beam element for linear elastic stress analysis of curved pipes under generalized in-plane loading. Comput Struct 1992;43(4):787-94.

[8] Melo FJMQ, Castro PMST. The linear elastic stress analysis of curved pipes under generalized loads using a reduced integration finite ring element. J Strain Anal 1997:32(1):47-59.

[9] Flügge W. Thin elastic shells. Berlin: Springer; 1973.

[10] Kitching R. Smooth and mitred pipe bends. In: Gill SS, editor. The stress analysis of pressure vessels and pressure vessels components. Oxford: Pergamon Press; 1970 [chapter 7] 